\title{
氮杂环卡宾双核金络合物催化的胺芳基化反应
}

\author{
张点 ${ }^{a}$ \\ ( ${ }^{a}$ 先进材料和精细化学品重点实验室 \\ 徐琴*,a施敏 $*, a, b$ \\ $\left({ }^{b}\right.$ 金属有机化学国家重点实验室 \\ 化学与分子工程学院 华东理工大学 上海 200237) \\ 中国科学院上海有机化学研究所 上海 200032)
}

\begin{abstract}
摘要 从联荎胺出发合成了氮杂环卡宾双核和单核金络合物, 通过 $\mathrm{X}$ 射线的单晶衍射确定了它们的结构, 并将其应用 于催化胺芳基化反应中，以高达 $95 \%$ 的收率得到吡咯烷类化合物. 综合上述实验结果，发现氮杂环卡宾双核金络合物 4b 中存在着 $\mathrm{Au}(\mathrm{I})-\mathrm{Au}(\mathrm{I})$ 间相互弱作用力, 而且这种弱相互作用可能对该催化反应起重要的作用, 以高收率得到吡咯烷 类化合物.
\end{abstract}

关键词 联荎胺; 氮杂环卡宾; 金络合物; 金-金相互作用; 胺芳基化

\section{N-Heterocyclic Carbene (NHC) Binuclear Gold Complexes Catalyzed Aminoarylation of Olefins}

\author{
Zhang, Rui ${ }^{a} \quad \mathrm{Xu}, \mathrm{Qin}^{*, a} \quad$ Shi, Min*,a,b \\ ( ${ }^{a}$ Key Laboratory for Advanced Materials and Institute of Fine Chemicals, School of Chemistry \& Molecular \\ Engineering, East China University of Science and Technology, Shanghai 200237, China) \\ ( ${ }^{b}$ State Key Laboratory of Organometallic Chemistry, Shanghai Institute of Organic Chemistry, Chinese Academy \\ of Sciences, Shanghai 200032, China)
}

\begin{abstract}
N$-Heterocyclic carbene (NHC) binuclear gold complexes and mononuclear gold complex have been successfully prepared from 1,1'-binaphthalenyl-2,2'-diamine (BINAM) in good yields. Their structures have been unambiguously determined by spectroscopic data and X-ray diffraction. On the basis of X-ray diffraction, a weak interaction between Au-Au has been identified in the $N$-heterocyclic carbene (NHC) binuclear gold complex $\mathbf{4 b}$, in which the distance of $\mathrm{Au}(\mathrm{I})-\mathrm{Au}(\mathrm{I})$ is $4.190 \AA$. We also found that NHC-binuclear gold complex $\mathbf{4 b}$ is a more effective catalyst than that of NHC-mononuclear gold complex in the aminoarylation of olefins under identical conditions. Based on these experimental data, the improved yield of aminoarylation is possibly attributed to the weak $\mathrm{Au}(\mathrm{I})-\mathrm{Au}(\mathrm{I})$ interaction in the $N$-heterocyclic carbene (NHC) binuclear gold complex $\mathbf{4 b}$. A plausible reaction mechanism has been proposed on the basis of previous literature. The reaction procedure is quite simple. When NHC-gold(I) catalyst $\mathbf{4 b}(3 \mathrm{~mol} \%, 3.0 \mu \mathrm{mol})$ was dissolved in solvent $(2.0 \mathrm{~mL})$ in a flame-dried Schlenk tube equipped with a septum cap and stirring bar, the additive $\operatorname{AgSbF}_{6}(6.0 \mu \mathrm{mol})$ was added under argon, and then the mixture was stirred under argon at room temperature for $10 \mathrm{~min}$. Alkylamine $(0.1 \mathrm{mmol})$, arylboronic acid $(0.2 \mathrm{mmol})$, and selectfluor $(0.2 \mathrm{mmol})$ were added, and then the reaction mixture was stirred at $60{ }^{\circ} \mathrm{C}$ for $12 \mathrm{~h}$. The crude product was concentrated under reduced pressure, and purified by flash chromatography on silica gel (eluent: EtOAc/petroleum ether $=1 / 16$ ) to yield the pure corresponding product. Recently, a number of bis(gold) vinyl species have been isolated from homogeneous gold catalysis and have been identified as the key intermediate in the catalytic process. Meanwhile, binuclear gold complexes have been also realized as the key species in the homogeneous gold catalysis recently. In this paper, we first disclosed that the $\mathrm{N}$-heterocyclic carbene (NHC) binuclear gold complex has a weak interaction between $\mathrm{Au}(\mathrm{I})$ - $\mathrm{Au}(\mathrm{I})$, which plays an important role in the aminoarylation of olefins.

Keywords BINAM; $N$-heterocyclic carbene; gold complex; gold(I)-gold(I) interaction; aminoarylation
\end{abstract}

\section{1 引言}

在过去的几十年中, 第 VIII 族过渡金属催化的交叉 偶联反应在有机合成中被广泛应用 ${ }^{[1]}$. 这主要归功于
钯、铂及镍与有机分子间的氧化加成及还原消除能 力 $^{[2 \sim 9]}$. 近期, 金均相催化反应受到了广泛的关注，相关 的研究工作主要集中于 $\mathrm{Au}(\mathrm{I})$ 对底物中 $\pi$-键的活化能力 的研究 ${ }^{[10 \sim 17]}$. Hashmi ${ }^{[18]}$, Zhang ${ }^{[19 \sim 21]}$, Toste ${ }^{[22 \sim 25]}$ 及其他

\footnotetext{
*E-mail: mshi@mail.sioc.ac.cn; Tel.: 021-54925137; Fax: 021-64166128

Received June 5, 2012; published July 17, 2012.

Supporting information for this article is available free of charge via the Internet at http://sioc-journal.cn.

Project supported by the Shanghai Municipal Committee of Science and Technology (No. 11JC1402600), National Basic Research Program of China (973)-(No. 2009CB825300), the Fundamental Research Funds for the Central Universities and the National Natural Science Foundation of China (Nos. 21072206, 20472096, 20872162, 20672127, 21121062 and 20732008).

项目受上海市科委(No. 11JC1402600)、中国国家基础研究项目(973)-(No. 2009CB825300)、中央高校基本科研业务费和国家自然科学基金(Nos. 21072206, 20472096, 20872162, 20672127, 21121062 和 20732008)资助.
} 
小组 ${ }^{[26 ~ 31]}$ 发现了 $\mathrm{Au}(\mathrm{I}) / \mathrm{Au}(\mathrm{III})$ 催化的氧化还原循环体 系, 拓展了 $\mathrm{Au}(\mathrm{I})$ 的催化领域. 鉴于此, 金催化的有机化 学反应将可能成为继钯、铂及镍催化的交叉偶联反应后 又一研究热点.

金属一金属之间存在 $\mathrm{d}^{10}-\mathrm{d}^{10}$ 弱相互作用早在 1977 年 就已被发现 ${ }^{[32]}$, 此后对于 $\mathrm{Au}(\mathrm{I})-\mathrm{Au}(\mathrm{I})$ 弱相互作用的研究 被大量报道 ${ }^{[33,34]}$. 最近 Gagné 小组在研究联烯的异构化 反应中提出双金属 $\mathrm{Au}(\mathrm{I})-\mathrm{Au}(\mathrm{I})$ 之间存在弱的相互作用 可增强催化剂的立体选择性 ${ }^{[35]}$. Che 小组将具有 $\mathrm{Au}(\mathrm{I})-\mathrm{Au}(\mathrm{I})$ 弱相互作用的络合物成功地应用到联烯与吲 哚的分子间不对称氢芳基化反应中 ${ }^{[36]}$. Toste 小组最近 也报道了双核 $\mathrm{Au}(\mathrm{I})$-双膦配体催化烯烃的氮杂芳基化反 应, 发现了双核 $\mathrm{Au}(\mathrm{I})$-双膦配体络合物的催化效果优于 单核 $\mathrm{Au}(\mathrm{I})$ 络合物, 并首次提出双金属中心促进的胺芳 基化反应催化循环机理 ${ }^{[37]}$.

氮杂环卡宾配体与膦配体相比是一类强的 $\sigma$-供电 子和弱的 $\pi$-受体 ${ }^{[38,39]}$. 大多数金属氮杂环卡宾络合物是 对空气、湿气不敏感的化合物, 因其操作十分方便而被 广泛研究 ${ }^{[40 ~ 42]}$. 此前我们小组合成了一系列基于联菜 胺和 $\mathrm{H}_{8}$-联萗胺骨架的氮杂环卡宾金属络合物, 并成功 应用到许多催化反应中 ${ }^{[43 \sim 56]}$

通过查阅文献, 氮杂环卡宾双核 $\mathrm{Au}(\mathrm{I})$ 络合物是否 存在 $\mathrm{Au}(\mathrm{I})-\mathrm{Au}(\mathrm{I})$ 弱相互作用, 并且这种弱相互作用能否 有效促进催化反应还未见报道. 在此, 我们合成了基于 联荎骨架的氮杂环卡宾双核和单核 $\mathrm{Au}(\mathrm{I})$ 络合物, 通过 $X$ 光单晶衍射确定其结构, 研究氮杂环卡宾双核 $\mathrm{Au}(\mathrm{I})$ 络合物是否存在 $\mathrm{Au}(\mathrm{I})-\mathrm{Au}(\mathrm{I})$ 弱相互作用; 并且进一步将 其应用到催化胺芳基化反应中, 希望确认 $\mathrm{Au}(\mathrm{I})-\mathrm{Au}(\mathrm{I})$ 弱 相互作用对于催化反应是否效果更好.

\section{2 结果与讨论}

\section{1 氮杂环卡宾金催化剂的合成}

参照我们小组先前报道的合成方法, 我们合成了氮 杂环卡宾金络合物 $4 a \sim 4 b$ 和 6 (如图 1). 其中氮杂环卡 宾金络合物 $4 \mathbf{a}$ 为已知化合物 ${ }^{[57]}$, 氮杂环卡宾金络合物 $\mathbf{4} \mathbf{b}^{[58]}$ 与 $\mathbf{6}^{[59]}$ 的结构通过 $\mathrm{X}$ 光单晶衍射得以确认(详见图 2, 图 3).

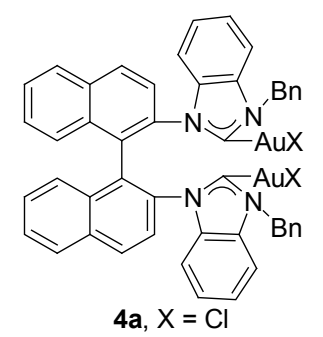

4b, $X=B r$

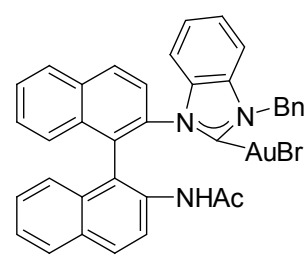

6
图 1 氮杂环卡宾金络合物 $4 a \sim 4 b$ 和 6

Figure 1 NHC-gold(I) complexes $\mathbf{4 a} \sim \mathbf{4 b}$ and $\mathbf{6}$

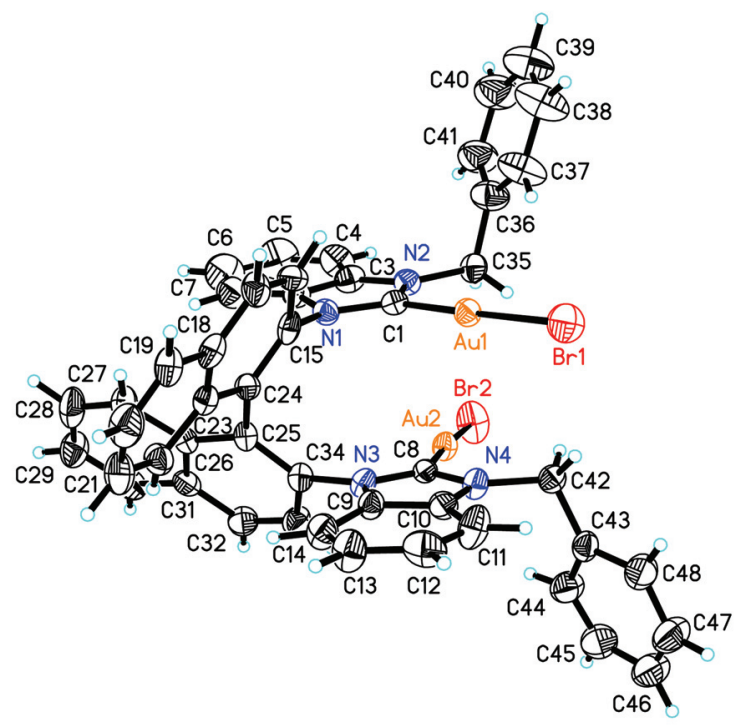

图 2 氮杂环卡宾双核金络合物 $4 b$

Figure 2 ORTEP drawing of NHC-gold(I) complex $\mathbf{4 b}$ with thermal ellipsoids at the $30 \%$ probability level. Selected bond distances $(\AA)$ and angles (deg): $\mathrm{Au}(1)-\mathrm{C}(1)$ 1.967(8), $\mathrm{Au}(1)-\mathrm{Br}(1)$ 2.3756(11), $\mathrm{Au}(2)-\mathrm{C}(8)$ 1.984(7), $\mathrm{Au}(2)-\mathrm{Br}(2) \quad 2.3725(11), \quad \mathrm{C}(1)-\mathrm{Au}(1)-\mathrm{Br}(1)$ 177.1(2), $\mathrm{C}(8)-$ $\mathrm{Au}(2)-\mathrm{Br}(2)$ 176.4(2), N(1)-C(1)-N(2) 104.9(7), N(3)-C(8)-N(4) 107.2(6)

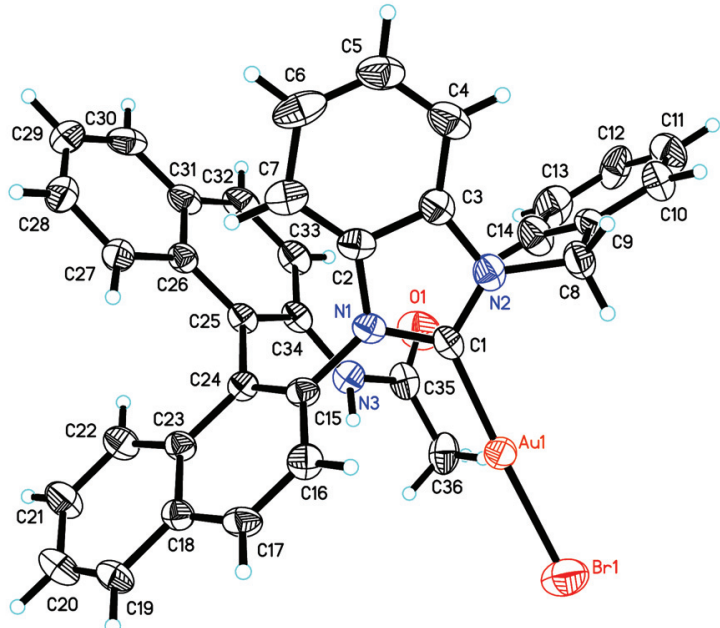

图 3 氮杂环卡宾金络合物 6

Figure 3 ORTEP drawing of NHC-gold(I) complex 6 with thermal ellipsoids at the $30 \%$ probability level. Selected bond distances $(\AA)$ and angles (deg): $\mathrm{Au}(1)-\mathrm{C}(1)$ 2.016(7), $\mathrm{Au}(1)-\mathrm{Br}(1)$ 2.3621(11), $\mathrm{O}(1)-\mathrm{C}(35)$ 1.215(9), $\mathrm{N}(3)-\mathrm{C}(35) \quad 1.353(10), \mathrm{C}(1)-\mathrm{Au}(1)-\mathrm{Br}(1) \quad 177.84(18), \mathrm{N}(3)-$ $\mathrm{C}(35)-\mathrm{O}(1) \quad 122.8(8), \quad \mathrm{N}(1)-\mathrm{C}(1)-\mathrm{N}(2) \quad 107.7(7), \quad \mathrm{O}(1)-\mathrm{C}(35)-\mathrm{C}(36)$ $121.0(8)$

\section{2 胺芳基化反应的条件优化}

首先, 我们将磺酰胺 $7 \mathbf{a}(0.1 \mathrm{mmol})$ 作为底物, selectfluor $9(0.2 \mathrm{mmol})$ 作为氧化剂, 在氮杂环卡宾金络合 物催化下与苯基硼酸 $8 \mathrm{a}(0.2 \mathrm{mmol})$ 发生胺芳基化反应, 来考察各类金催化剂的催化性能. 反应结果显示, 等量 的氮杂环卡宾金络合物 $4 \mathrm{~b}$ 比 $4 \mathrm{a}$ 和 6 具有更高的催化活 性，而且比 2 倍量的氮杂环卡金络合物 6 催化活性还高 
(表 1, Entries 1 3, 20). 进一步从单晶数据上分析, 氮杂 环卡宾金络合物 $4 \mathbf{a}$ 中 $\mathrm{Au}(\mathrm{I})-\mathrm{Au}(\mathrm{I})$ 之间的距离为 $5.793 \AA$, 氮杂环卡宾金络合物 $4 b$ 中 $\mathrm{Au}(\mathrm{I})-\mathrm{Au}(\mathrm{I})$ 之间的距离为 $4.190 \AA$, 而金原子的范德华半径为 $1.66 \sim 2.43 \AA^{[60]}$, 因 此我们推测氮杂环卡宾金络合物 $4 \mathbf{b}$ 可能 $\mathrm{Au}(\mathrm{I})-\mathrm{Au}(\mathrm{I})$ 间 存在相互弱作用力, 而且这种弱相互作用对该催化反应 起重要的作用. 遗憾的是我们目前无法获悉 $\mathrm{Au}(\mathrm{III})$ 活性 物种的结构, 在现阶段还不能给出明确的结论. 使用光 学纯的催化剂 4b 催化该反应, 以 $84 \%$ 的收率得到外消 旋的目标产物 10a. 接着, 我们对氧化剂的用量进行了 优化, 增加氧化剂的用量会降低产物 10a 的收率(表 1 , Entries 4 5). 然后, 我们对反应的添加剂进行了笁选, 发现以 $\mathrm{AgSbF}_{6}$ 作为添加剂, 可以大大加快反应速率, 反

表 1 反应条件优化

Table 1 Optimization of reaction conditions

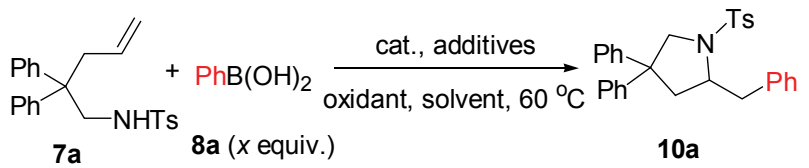

\begin{tabular}{|c|c|c|c|c|c|c|c|}
\hline Entry $^{a}$ & Cat. & $x$ & Additive & Oxidant & Solvent & Time/h & Yield $^{b}$ \\
\hline 1 & $4 a$ & 2.0 & - & 9 (2.0 equiv.) & $\mathrm{CH}_{3} \mathrm{CN}$ & 48 & 46 \\
\hline 2 & $4 b$ & 2.0 & - & 9 (2.0 equiv.) & $\mathrm{CH}_{3} \mathrm{CN}$ & 48 & 85 \\
\hline $3^{c}$ & 6 & 2.0 & - & 9 (2.0 equiv.) & $\mathrm{CH}_{3} \mathrm{CN}$ & 48 & 80 \\
\hline 4 & $4 b$ & 2.0 & - & 9 (3.0 equiv.) & $\mathrm{CH}_{3} \mathrm{CN}$ & 48 & 36 \\
\hline 5 & $4 b$ & 2.0 & - & 9 (4.0 equiv.) & $\mathrm{CH}_{3} \mathrm{CN}$ & 48 & 25 \\
\hline 6 & $4 b$ & 2.0 & $\mathrm{AgBF}_{4}$ & 9 (2.0 equiv. $)$ & $\mathrm{CH}_{3} \mathrm{CN}$ & 12 & trace \\
\hline 7 & $4 b$ & 2.5 & $\mathrm{AgOTf}$ & 9 (2.0 equiv.) & $\mathrm{CH}_{3} \mathrm{CN}$ & 12 & trace \\
\hline 8 & $4 b$ & 2.0 & $\mathrm{AgOTs}$ & 9 (2.0 equiv.) & $\mathrm{CH}_{3} \mathrm{CN}$ & 12 & trace \\
\hline 9 & $4 b$ & 2.0 & $\mathrm{AgOAc}$ & 9 (2.0 equiv.) & $\mathrm{CH}_{3} \mathrm{CN}$ & 12 & 40 \\
\hline 10 & $4 b$ & 2.0 & $\mathrm{AgSbF}_{6}$ & 9 (2.0 equiv.) & $\mathrm{CH}_{3} \mathrm{CN}$ & 12 & 84 \\
\hline 11 & $4 b$ & 2.0 & $\mathrm{AgCOCF}_{3}$ & 3 ( $(2.0$ equiv.) & $\mathrm{CH}_{3} \mathrm{CN}$ & 12 & 50 \\
\hline 12 & $4 b$ & 2.0 & $\mathrm{AgSbF}_{6}$ & 9 (2.0 equiv. $)$ & toulene & 12 & n.r..$^{d}$ \\
\hline 13 & $4 b$ & 2.0 & $\mathrm{AgSbF}_{6}$ & 9 (2.0 equiv.) & dioxane & 12 & n.r..$^{d}$ \\
\hline 14 & $4 b$ & 2.0 & $\mathrm{AgSbF}_{6}$ & 9 (2.0 equiv.) & $\mathrm{MeOH}$ & 12 & trace \\
\hline $15^{e}$ & $4 b$ & 2.0 & $\mathrm{AgSbF}_{6}$ & 9 (2.0 equiv.) & $\mathrm{CH}_{3} \mathrm{CN} / \mathrm{H}_{2} \mathrm{O}$ & 12 & 41 \\
\hline 16 & $4 b$ & 1.0 & $\mathrm{AgSbF}_{6}$ & 9 (2.0 equiv.) & $\mathrm{CH}_{3} \mathrm{CN}$ & 12 & trace \\
\hline 17 & $4 b$ & 1.5 & $\mathrm{AgSbF}_{6}$ & 9 (2.0 equiv.) & $\mathrm{CH}_{3} \mathrm{CN}$ & 12 & 70 \\
\hline 18 & $4 b$ & 3.0 & $\mathrm{AgSbF}_{6}$ & 9 (2.0 equiv.) & $\mathrm{CH}_{3} \mathrm{CN}$ & 12 & 20 \\
\hline 19 & $4 b$ & 4.0 & $\mathrm{AgSbF}_{6}$ & 9 (2.0 equiv) & $\mathrm{CH}_{3} \mathrm{CN}$ & 12 & 12 \\
\hline $20^{f}$ & 6 & & - & $\mathbf{9}$ (2.0 equiv) & $\mathrm{CH}_{3} \mathrm{CN}$ & 48 & 53 \\
\hline
\end{tabular}

${ }^{a}$ Reaction conditions: substrate $7 \mathbf{a}(0.1 \mathrm{mmol})$, phenylbronic acid $8 \mathbf{8 a}(0.2$ mmol), NHC-gold(I) complex 4 ( $3 \mathrm{~mol} \%, 3.0 \mu \mathrm{mol})$, additive, oxidant 9 ( $2 \sim 4$ equiv.) and solvent $(2.0 \mathrm{~mL})$ reacted at $60{ }^{\circ} \mathrm{C} ;{ }^{b}$ Isolated yields; ${ }^{c}$ Reaction conditions: substrate $7 \mathbf{a}(0.1 \mathrm{mmol})$, phenylbronic acid $(0.2 \mathrm{mmol})$, NHC-gold(I) complex 6 ( $6 \mathrm{~mol} \%, 6.0 \mu \mathrm{mol})$, oxidant $9(0.2 \mathrm{mmol})$ and solvent $(2.0 \mathrm{~mL})$ reacted at $60{ }^{\circ} \mathrm{C}$; ${ }^{d}$ No reaction; ${ }^{e} \mathrm{CH}_{3} \mathrm{CN} / \mathrm{H}_{2} \mathrm{O}=10: 1 .{ }^{f}$ Reaction conditions: substrate 7a $(0.1 \mathrm{mmol})$, phenylbronic acid $(0.2 \mathrm{mmol})$, NHC-gold(I) complex 6 (3 mol\%, $3.0 \mu \mathrm{mol})$, oxidant $9(0.2 \mathrm{mmol})$ and solvent $(2.0 \mathrm{~mL})$ reacted at $60{ }^{\circ} \mathrm{C}$.
应时间在相同条件下从原来的 $48 \mathrm{~h}$ 缩短至 $12 \mathrm{~h}$ ，以 $84 \%$ 的收率得到目标产物(表 1, Entries 2，6１1). 对溶剂笁 选的结果显示，乙腈是最佳选择(表 1, Entries 12 15). 最后，我们对苯基硼酸的用量也进行了考察，2 equiv.苯 基硼酸能以最高产率得到产物 10a, 无论增加还是减少 苯基硼酸的用量都会使反应产物收率降低(表 1, Entries 16 19).

\section{3 胺芳基化反应的底物拓展}

在最优条件下，我们考察了底物的普适性. 无论底 物 7 是供电子还是吸电子的苯磺酰基团时，此反应体系 都可以顺利地进行以较高的收率得到目标产物(表 2 , Entries 1 4). 偕二芳基/烷基作用对于此反应体系影响

表 2 底物拓展

Table 2 Aminoarylation of unactivated alkenes in the presence of NHC-gold(I) complex $\mathbf{4 b}$

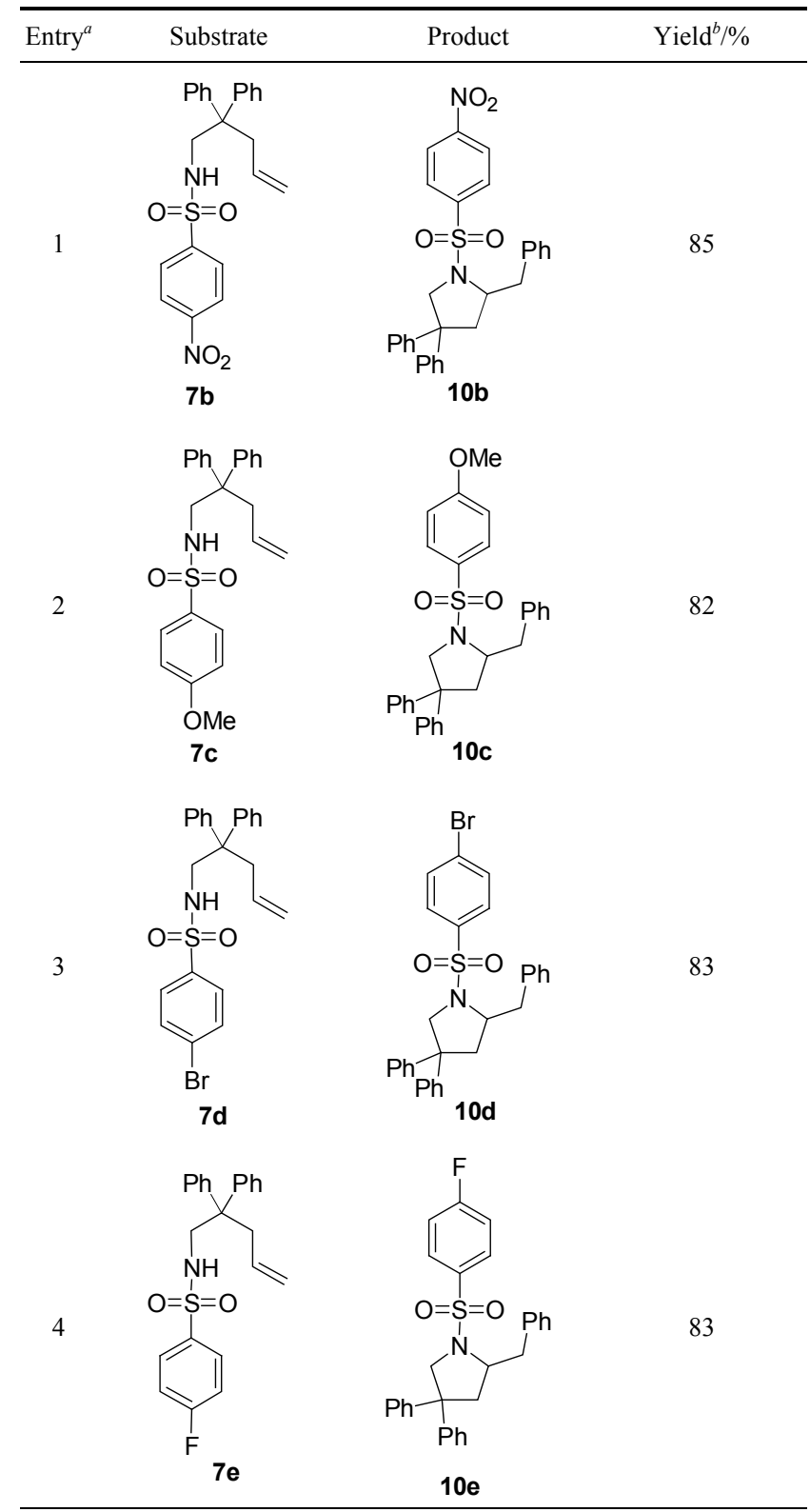




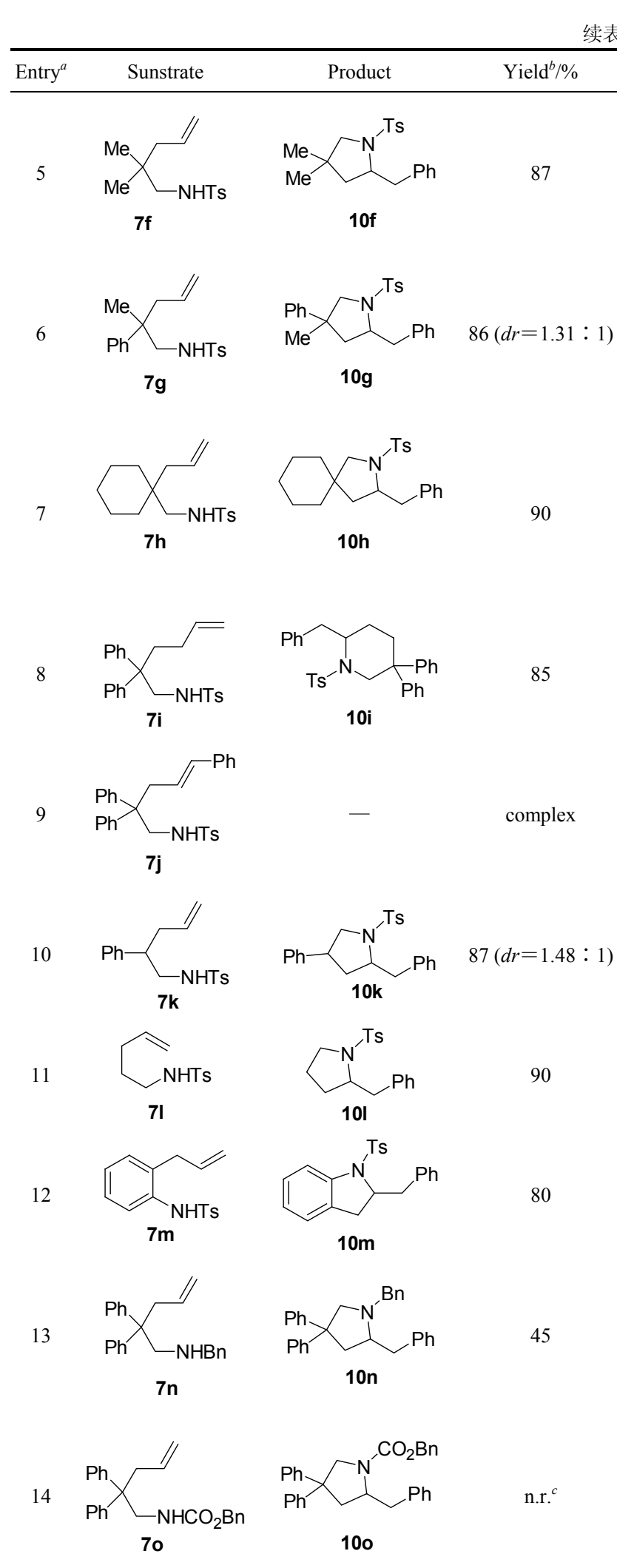

${ }^{a}$ Reaction conditions: substrate $7(0.1 \mathrm{mmol})$, phenylboronic acid $(0.2 \mathrm{mmol})$, NHC-gold(I) complex $4 \mathbf{b}(3 \mathrm{~mol} \%, 3.0 \mu \mathrm{mol}), \mathrm{AgSbF}_{6}(6.0 \mu \mathrm{mol})$, selectfluor $9(0.2 \mathrm{mmol})$ and $\mathrm{CH}_{3} \mathrm{CN}(2.0 \mathrm{~mL})$ reacted at $60{ }^{\circ} \mathrm{C} ;{ }^{b}$ Isolated yields; ${ }^{c}$ No reaction.

不大(表 2, Entries 5 7, 10 11). 哌啶类化合物 10i 也可 以以 $85 \%$ 的收率顺利得到(表 2 , Entry 8 ). 值得欣喜地是
我们可以在温和的条件下得到官能团化的 2,3-二氢吲哚 类化合物 $\mathbf{1 0 m}$ (表 2, Entry 12). 另外，底物 7 中氮原子的 保护基为供电子苠基时，反应也可发生，但反应的收率 会骤然下降至 45\% (表 2, Entry 13). 当 $\mathrm{N}$ 原子上含有 $\mathrm{CO}_{2} \mathrm{Bn}$ 保护基时, 此反应不能发生(表 2, Entry 14). 当底 物 7j 中烯烃的端位有苯基取代时, 该催化体系也不适用 (表 2, Entry 9).

\section{4 芳基硼酸的适用范围}

在最优条件下，无论是贫电子还是富电子的芳基硼 酸与底物 7 反应都可以顺利反应得到目标产物(表 3 , Entries $1 \sim 4$, and $6 \sim 7$ ). 当底物 8 为萗碀酸时, 没有得到 目标产物(表 3, Entries 5 and 8).

表 3 芳基嗍酸的适用范围

Table 3 Boronic acid scope for the aminoarylation

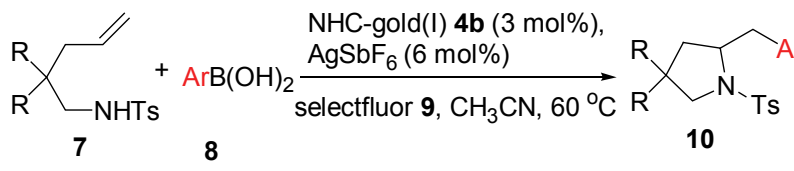

\begin{tabular}{|c|c|c|c|}
\hline Entry $^{a}$ & Substrate & $\operatorname{ArB}(\mathrm{OH})_{2}$ & Yield $^{b} \%$ \\
\hline 1 & $7 \mathbf{a}, \mathrm{R}=\mathrm{Ph}$ & $\mathbf{8 b}, \mathrm{Ar}=o-\mathrm{Me}-\mathrm{C}_{6} \mathrm{H}_{4}$ & $10 p, 45$ \\
\hline 2 & $7 \mathbf{a}, \mathrm{R}=\mathrm{Ph}$ & $\mathbf{8 c}, \mathrm{Ar}=m-\mathrm{Me}-\mathrm{C}_{6} \mathrm{H}_{4}$ & 10q, 85 \\
\hline 3 & $7 \mathbf{a}, \mathrm{R}=\mathrm{Ph}$ & $\mathbf{8 d}, \mathrm{Ar}=p-\mathrm{Me}-\mathrm{C}_{6} \mathrm{H}_{4}$ & $10 \mathrm{r}, 70$ \\
\hline 4 & $7 \mathbf{a}, \mathrm{R}=\mathrm{Ph}$ & $\mathbf{8 e}, \mathrm{Ar}=p-\mathrm{Cl}-\mathrm{C}_{6} \mathrm{H}_{4}$ & 10s, 88 \\
\hline 5 & $7 \mathbf{a}, \mathrm{R}=\mathrm{Ph}$ & $\mathbf{8 f}, \mathrm{Ar}=2$-naphthyl & trace \\
\hline 6 & $7 \mathrm{l}, \mathrm{R}=\mathrm{H}$ & $\mathbf{8 b}, \mathrm{Ar}=o-\mathrm{Me}-\mathrm{C}_{6} \mathrm{H}_{4}$ & 10t, 95 \\
\hline 7 & $71, \mathrm{R}=\mathrm{H}$ & $\mathbf{8 g}, \mathrm{Ar}=p-\mathrm{F}-\mathrm{C}_{6} \mathrm{H}_{4}$ & $10 u, 90$ \\
\hline 8 & $7 \mathbf{l}, \mathrm{R}=\mathrm{H}$ & $\mathbf{8 f}, \mathrm{Ar}=2$-naphthyl & trace \\
\hline
\end{tabular}

\section{5 反应机理}

在 Zhang ${ }^{[20]}$ 和 Toste ${ }^{[23,37]}$ 小组研究基础上，我们提出 了此反应的机理(如图 4). 首先氮杂环卡宾双核金(I)络 合物 4 被 selectfluor 9 氧化生成金(III)络合物 11, 然后金 (III)络合物 11 与底物 7a 中的烯烃配位，分子内的胺亲 核进攻双键生成中间体化合物 12, 化合物 12 中的氟原 子再与芳基嗍酸中的硼原子相互作用, 双分子还原消除 后得到目标产物 $\mathbf{1 0 .}$

\section{3 结论}

本文作者从联萗胺出发合成了一些氮杂环卡宾金 络合物，将其成功应用到胺芳基化反应中，以高达 95\% 的收率得到吡咯烷类化合物. 通过比较 $\mathrm{Au}(\mathrm{I})-\mathrm{Au}(\mathrm{I})$ 间的 距离和 $\mathrm{Au}$ 原子的范德华半径, 推断催化剂 $\mathbf{4 b}$ 中可能存 在 $\mathrm{Au}(\mathrm{I})-\mathrm{Au}(\mathrm{I})$ 弱作用力, 而且这种弱相互作用可能对该 催化反应起重要的作用. 


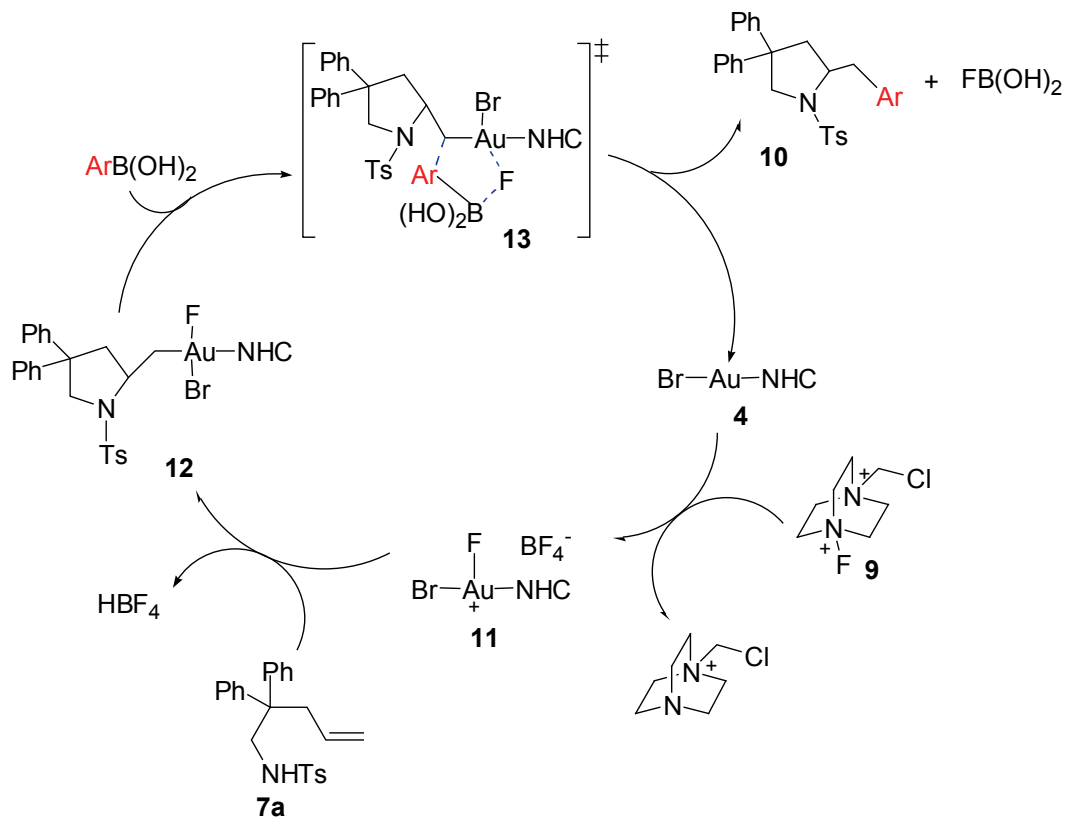

图 4 反应机理

Figure 4 Proposed reaction mechanism for the gold-catalyzed aminoarylation reaction

\section{References}

[1] Nicolaou, K. C.; Bulger, P. G.; Sarlah, D. Angew. Chem., Int. Ed. $\mathbf{2 0 0 5}, 44,4442$.

[2] Shapiro, N. D.; Toste, F. D. Synlett 2010, 675.

[3] Fürstner, A. Chem. Soc. Rev. 2009, 38, 3208.

[4] Jiménez-Núñez, E.; Echavarren, A. M. Chem. Rev. 2008, 108, 3326.

[5] Shen, H. C. Tetrahedron 2008, 64, 7847.

[6] Gorin, D. J.; Sherry, B. D.; Toste, F. D. Chem. Rev. 2008, 108, 3351.

[7] Li, Z.; Brouwer, C.; He, C. Chem. Rev. 2008, 108, 3239.

[8] Arcadi, A. Chem. Rev. 2008, 108, 3266.

[9] Hashmi, A. S. K. Chem. Rev. 2007, 107, 3180.

[10] Teles, J. H.; Brode, S.; Chabanas, M. Angew. Chem., Int. Ed. 1998, $37,1415$.

[11] Mizushima, E.; Sato, K.; Hayashi, T.; Tanaka, M. Angew. Chem., Int. Ed. 2002, 41, 4563 .

[12] Reetz, M. T.; Sommer, K. Eur. J. Org. Chem. 2003, 3485.

[13] Mizushima, E.; Hayashi, T.; Tanaka, M. Org. Lett. 2003, 5, 3349.

[14] Nieto-Oberhuber, C.; Munuz, M. P.; Bunuel, E.; Nevado, C.; Cardenas, D. J.; Echavarren, A. M. Angew. Chem., Int. Ed. 2004, 43, 2403.

[15] Kennedy-Smith, J. J.; Staben, S. T.; Toste, F. D. J. Am. Chem. Soc. 2004, 126, 4526.

[16] Mamane, V.; Gress, T.; Krause, H.; Fürstner, A. J. Am. Chem. Soc. 2004, 126, 8654

[17] Luzung, M. R.; Markham, J. P.; Toste, F. D. J. Am. Chem. Soc. 2004, 126, 10858.

[18] Hashmi, S. K.; Ramamurthi, T. D.; Rominger, F. J. Organomet. Chem. 2009, 694, 592.

[19] Zhang, G. Z.; Peng, Y.; Cui, L.; Zhang, L. Angew. Chem., Int. Ed. 2009, $48,3112$.

[20] Zhang, G. Z.; Cui, L.; Wang, Y. Z.; Zhang, L. J. Am. Chem. Soc. 2010, 132, 1474 .

[21] Zhang, G.; Luo, Y.; Wang, Y.; Zhang, L. Angew. Chem., Int. Ed. 2011, 50, 4450.

[22] Melhado, A. D.; Brenzovich, W. E.; Lackner, A. D.; Toste, F. D. J. Am. Chem. Soc. 2010, 132, 8885 .

[23] Brenzovich, W. E.; Benitez, D.; Lackner, A. D.; Shunatona, H. P.; Tkatchouk, E.; Goddard, W. A.; Toste, F. D. Angew. Chem., Int. Ed. 2010, $49,5519$.

[24] Mankad, N. P.; Toste, F. D. J. Am. Chem. Soc. 2010, 132, 12859.

[25] Brenzovich, W. E.; Brazeau, J. F.; Toste, F. D. Org. Lett. 2010, 12, 4728.

[26] Ball, L. T.; Green, M.; Lloyd-Jones, G. C.; Russel, C. A. Org. Lett.
2010, 12, 4724.

[27] Wang, W.; Jasinski, J.; Hammond, G. B.; Xu, B. Angew. Chem., Int Ed. 2010, 49, 7247.

[28] Hopkinson, M. N.; Tessier, A.; Salisbury, A.; Giuffedi, G. T.; Combettes, L. E.; Gee, A. D.; Gouverneur, V. Chem. Eur. J. 2010, 16, 7443.

[29] de Haro, T.; Nevado, C. Angew. Chem., Int. Ed. 2011, 50, 906.

[30] Engle, K. M.; Mei, T.-S.; Wang, X.; Yu, J.-Q. Angew. Chem., Int. Ed. 2011, 50, 1478

[31] Hopkins, M. N.; Gee, A. D.; Gouverneur, V. Chem. Eur. J. 2011, 30, 8248.

[32] Avdeef, A.; Fackler Jr, J. P. Inorg. Chem. 1978, 17, 2182.

[33] Schmidbaur, H.; Schier, A. Chem. Soc. Rev. 2008, 37, 1931.

[34] Gimeno, M. C.; Laguna, A. Chem. Soc. Rev. 2008, 37, 1952.

[35] Tarselli, M. A.; Chianese, A. R.; Lee, S. J.; Gagné, M. R. Angew. Chem., Int. Ed. 2007, 46, 6670.

[36] Wang, M.-Z.; Zhou, C.-Y.; Guo, Z.; Wong, E. L.-M.; Wong, M.-K.; Che, C.-M. Chem. Asian J. 2011, 6, 812.

[37] Tkatchouk, E.; Mankad, N. P.; Benitez, D.; Goddard, W. A.; Toste, F. D. J. Am. Chem. Soc. 2011, 133, 14293.

[38] Lee, M.-T.; Hu, C.-H. Organometallics 2004, 23, 976.

[39] Huang, J.; Jafarpour, L.; Hillier, A. C.; Stevens, E. D.; Nolan, S. P. Organometallics 2001, 20, 2878.

[40] Bugaut, X.; Liu, F.; Glorius, F. J. Am. Chem. Soc. 2011, 133, 8130.

[41] Dabrowski, J. A.; Gao, F.; Hoveyda, A. H. J. Am. Chem. Soc. 2011, $133,4778$.

[42] Xiao, Y.-P.; Liu, X.-Y.; Che, C.-M. Angew. Chem., Int. Ed. 2011, $50,4937$.

[43] Xu, Q.; Duan, W.-L.; Lei, Z.-Y.; Zhu, Z.-B.; Shi, M. Tetrahedron 2005, 61, 11225.

[44] Chen, T.; Jiang, J.-J.; Xu, Q.; Shi, M. Org. Lett. 2007, 9, 865.

[45] Zhang, T.; Shi, M. Chem. Eur. J. 2008, 14, 3759-3764.

[46] Ma, G.-N.; Zhang, T.; Shi, M. Org. Lett. 2009, 11, 875-878.

[47] Wang, W.; Zhang, T.; Shi, M. Organometallics 2009, 28, 2640.

[48] Liu, Z.; Shi, M. Tetrahedron 2010, 66, 2619.

[49] Liu, Z.; Shi, M. Organometallics 2010, 29, 2831.

[50] Xu, Q.; Zhang, R.; Zhang, T.; Shi, M. J. Org. Chem. 2010, 75, 3935.

[51] Zhang, R.; Xu, Q.; Zhang, X.; Zhang, T.; Shi, M. Tetrahedron: Asymmetry 2010, 21, 1928.

[52] Wang, W.; Zhang, T.; Wang, F.; Shi, M. Tetrahedron 2011, 67, 1523.

[53] Liu, Z.; Gu, P.; Shi, M. Org. Lett. 2011, 13, 2314.

[54] Liu, Z.; Gu, P.; Shi, M. Chem. Eur. J. 2011, 17, 5796.

[55] Wang, W.; Yang, J.; Wang, F.; Shi, M. Organometallics 2011, 30, 3859 . 
[56] Zhang, R.; Xu, Q.; Mei, L.; Li, S.; Shi, M. Tetrahedron 2012, 68, 3172 .

[57] Yang, J.; Zhang, R.; Wang, W.; Zhang, Z.; Shi, M. Tetrahedron: Asymmetry 2012, 22, 2029.

[58] The crystal data of $\mathbf{4 b}$ have been deposited in CCDC with number 870331. Empirical Formula: $\mathrm{C}_{48} \mathrm{H}_{34} \mathrm{Au}_{2} \mathrm{Br}_{2} \mathrm{~N}_{4}$; Formula Weight: 1220.54; Crystal Color, Habit: colorless, Crystal Dimensions: 0.256 $\times 0.117 \times 0.105 \mathrm{~mm}$; Crystal System: Orthorhombic; Lattice Parameters: $a=10.2106(5) \AA, b=21.7203(11) \AA, c=23.1654(12) \AA, \alpha$ $=90^{\circ}, \quad \beta=90^{\circ}, \gamma=90^{\circ}, \quad V=5137.6(4) \AA^{3}$; Space group: $P 2(1) 2(1) 2(1) ; Z=4 ; D_{\text {calc }}=1.578 \mathrm{~g} / \mathrm{cm}^{3} ; F_{000}=2312$; Final $R$ indices $[I>2 \sigma(\mathrm{I})]: R_{1}=0.0404 ; w R_{2}=0.0977$.
[59] The crystal data of 6 have been deposited in CCDC with number 877599. Empirical Formula: $\mathrm{C}_{36} \mathrm{H}_{27} \mathrm{AuBrN}_{3} \mathrm{O}$; Formula Weight: 794.48; Crystal Color, Habit: colorless, Crystal Dimensions: 0.211 $\times 0.195 \times 0.164 \mathrm{~mm}$; Crystal System: Triclinic; Lattice Parameters: $a=9.1183(9) \AA, b=10.4649(10) \AA, c=15.5456(15) \AA, \alpha=$ $83.801(2)^{\circ}, \beta=79.355(2)^{\circ}, \gamma=86.758(2)^{\circ}, V=1448.3(2) \AA^{3}$; Space group: $P-1 ; Z=2 ; D_{\text {calc }}=1.822 \mathrm{~g} / \mathrm{cm}^{3} ; F_{000}=772$; Final $R$ indices $[I>2 \sigma(I)]: R_{1}=0.0462 ; w R_{2}=0.1140$.

[60] Hu, S.-Z.; Zhou, Z.-H.; Cai, Q.-R. Acta Phys.-Chim. Sin. 2003, 19 1073. (胡盛志, 周朝晖, 蔡启瑞, 物理化学学报, 2003, 19, 1073.)

(Zhao, X.) 